\title{
The impact of the extent of surgical resection on survival of gastric cancer patients
}

\author{
This article was published in the following Dove Press journal: \\ OncoTargets and Therapy \\ 28 July 2016 \\ Number of times this article has been viewed
}

\section{Kostadin Georgiev Angelov Mariela Borisova Vasileva \\ Konstantin Savov Grozdev Svetoslav Yordanov Toshev Manol Bonev Sokolov Georgi Todorov Todorov}

Department of Surgery, Medical University of Sofia, Aleksandrovska University Hospital, Sofia, Bulgaria

Correspondence: Kostadin Georgiev Angelov Department of Surgery, Medical University of Sofia, Aleksandrovska University Hospital, I Georgi Sofiiski Str, I43I Sofia, Bulgaria Email dr.k.angelov@gmail.com
Objective: The aim of this study was to examine the significance of the extent of gastric resection on the postoperative and overall gastric cancer survival.

Background: Resection with clean margins ( $4 \mathrm{~cm}$ or more) is widely accepted as the standardized goal for radical treatment of gastric cancer according to current guidelines, while the type of resection (subtotal or total) is still a matter of debate.

Patients and methods: The study included 155 patients diagnosed and treated in the Department of Surgery, Aleksandrovska University Hospital between January 2005 and December 2014. In order to determine the significance of the resection volume, we excluded from the study 54 patients receiving palliative intervention or staging exploratory laparoscopy. The remaining 101 patients were divided into two groups based on the volume of the performed gastric resection (total and subtotal) and compared based on overall survival and perioperative mortality. We also investigated the 3-year survival in the two groups as well as the overall survival only in the subgroup of patients with D2 lymphadenectomy.

Results: We could not determine any statistically significant difference in overall survival and 3 -year survival $(P=0.990)$ based on the extent of surgical resection $(P=0.824)$ or perioperative mortality. The statistical analysis on patients with D2 lymph node dissection only did not show significance for overall survival.

Conclusion: Our study shows no difference in safety and long-term survival rate of patients with gastric carcinoma based on the volume of stomach resection. Comparison with other studies also shows no difference in survival based on volume of the resection.

Keywords: gastric cancer, perioperative mortality, overall survival

\section{Introduction}

In 2012, there were nearly a million new cases of gastric cancer $(952,000$ cases or $6.8 \%$ of all cancer cases). Based on rate of occurrence, this has put gastric cancer in fifth place in the world. It is also the third leading cause of death in both sexes $(723,000$ deaths or $8.8 \%$ of all cancer deaths). ${ }^{1}$

Bulgaria (a country with an average gastric cancer risk rate) is in sixth place in males' rate of occurrence and eighth among females. This makes up to $4.9 \%$ of all malignant diseases. The standardized morbidity and mortality of patients with gastric cancer in Bulgaria are higher than the average for Europe. The 5-year relative gastric cancer survival rate in Bulgaria is $10.2 \%$ for males and $13.7 \%$ for females, with a total of $11.8 \%$ for both sexes. This is lower than the average number for Europe $-25.1 \%{ }^{2}$

According to the Japanese Association of Gastric Cancer, the standard surgical procedure for clinically positive lymph nodes or T2-T4 tumors is either a total gastrectomy (TG) or a distal gastrectomy. The distal gastrectomy is the method of choice only when the surgeons can achieve clean proximal margins. ${ }^{3}$ 
According to the review on the update of gastric cancer treatment by the Chinese Medical Association, 2014, ${ }^{4}$ recommended surgery for gastric cancer is a standard gastrectomy, which is comprised of TG and distal subtotal gastrectomy (STG) for clinically node-positive or T2-4a tumors. Some patients with clinical $\mathrm{T} 1$ and $\mathrm{N} 0$ can receive modified gastric resection according to the tumor location.

According to the last revision of the National Comprehensive Cancer Network - Version 3, 2015, surgery is considered the primary treatment for gastric cancer. Resection with clean margins ( $4 \mathrm{~cm}$ or more) is widely accepted as the standardized goal, while the type of resection (subtotal or total) and the volume of lymph dissection are still a matter of debate. ${ }^{5}$

STG is the preferred method for distal carcinoma. This procedure leads to similar results as the TG but has significantly less postoperative complications. The proximal gastrectomy and the TG are both used for proximal carcinomas and are associated with postoperative diet imbalance.

According to European Society for Medical Oncology ${ }^{6}$ recommendations, the radical gastrectomy is best used for the resectable stage IB-III of the disease. The STG can be performed if the macroscopic proximal boundary of $5 \mathrm{~cm}$ can be achieved between the tumor and the esophagogastric junction. A resection line of $8 \mathrm{~cm}$ can be considered with different types of carcinoma. In the rest of the cases, they suggest the use of TG.

In 2014, based on the American College of Surgeons National Surgical Quality Improvement Program database, Bartlett et al reported a mortality rate of $4.7 \%$ within 30 days of TG for gastric malignancy. The authors found four significant preoperative prognostic factors that were associated with mortality: age $>70$ years, weight loss, ALB level, and pancreatectomy.?

The aim of this study is to examine the influence of the volume of the gastric resection on the postoperative and overall gastric cancer survival among patients in Bulgaria.

\section{Patients and methods}

\section{Patients}

In this study, we have included patients diagnosed and treated in Aleksandrovska University Hospital for the time period between January 2005 and December 2013. Approval from the ethical committee of the Department of Surgery at the University Hospital "Aleksandrovska" was obtained. The study only included patients who had signed an informed consent. We gathered information from the medical records about patients' sex, age at diagnosis, process localization, and the surgical intervention performed. The study included 155 patients -99 males and 56 females with an average age of 66.22 years (ranging from 27 to 85 years). They all entered the clinic randomly. Four different surgeons took part in this study. Each patient's condition was followed-up until January 31, 2014.

\section{Study design}

The design of the study is shown in Figure 1.

To determine the significance of the resection volume, we excluded from the study 54 patients receiving palliative intervention (gastroenteroanastomosis) or staging laparoscopy (Figure 1).

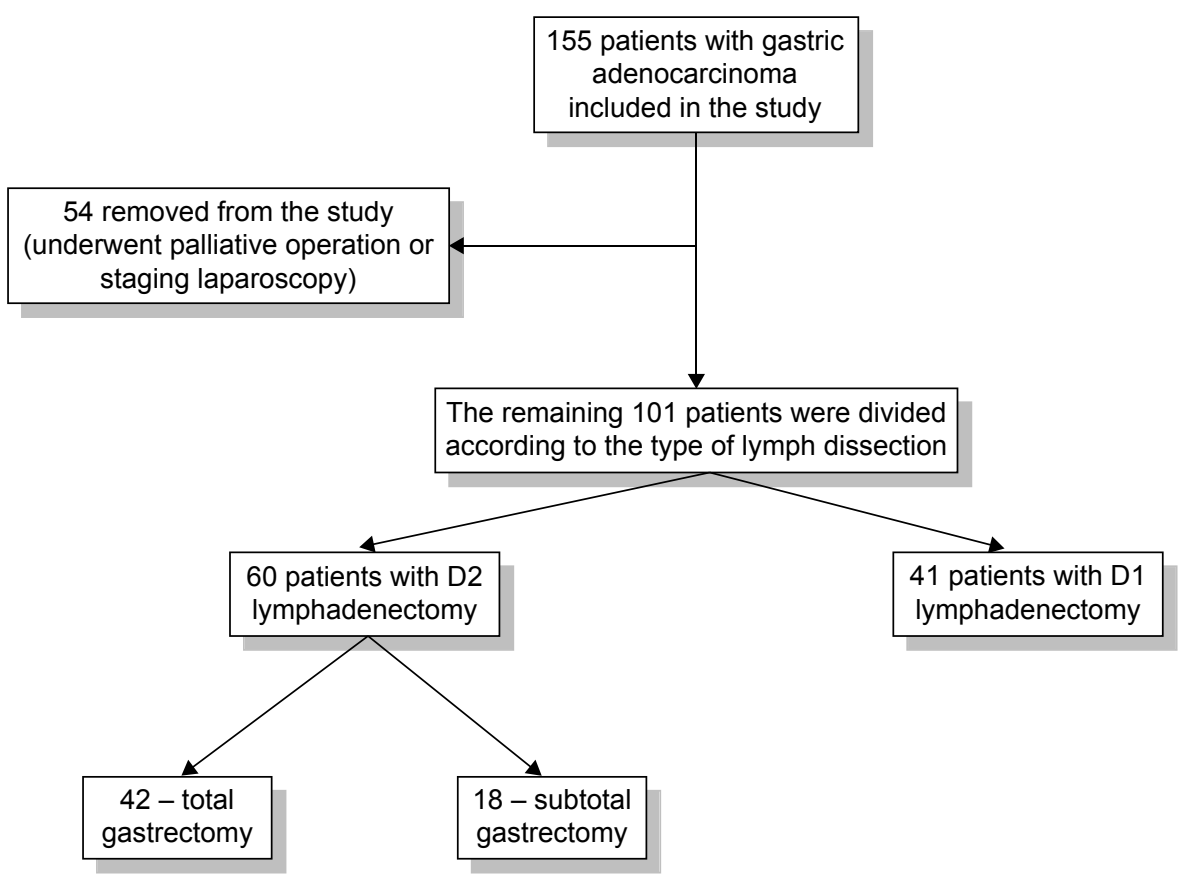

Figure I Study design. 
Total gastrectomy

Gastroenteroanastomosis

- Subtotal gastrectomy

Explorative laparotomy

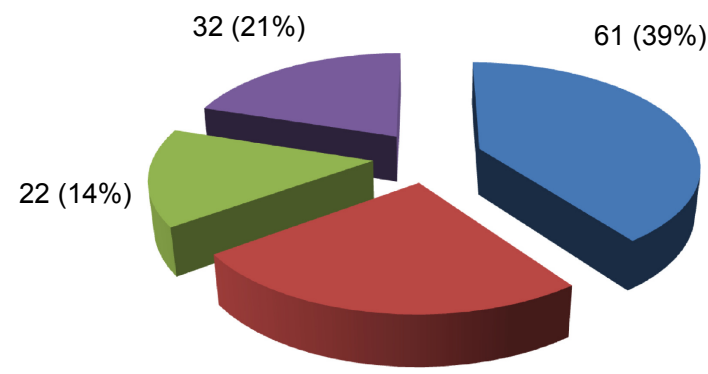

$40(26 \%)$

Figure 2 Patient groups based on the type of operation performed, n (\%)

The remaining 101 patients were divided into two groups based on the volume of the performed gastric resection - total or subtotal (Figure 2). The two groups were compared based on overall survival. In order to eliminate the influence of the insufficient time to follow those patients who were operated on recently and were still included in the study, we investigated the 3-year survival as well.

In the end, in order to exclude the influence of the volume of the lymph dissection on the overall survival (a factor with a proven value), from the 101 patients, we excluded those with a lymph dissection volume smaller than D2. We studied the significance of gastric resection on the overall survival in a subgroup of 60 patients with D2 lymphadenectomy.

\section{Statistical methods}

For calculating the data in the study, we used SPSS for Windows 13.0 (SPSS Inc., Chicago, IL, USA). We investigated the significance of the volume of gastric resection of 101 patients in overall survival using Kaplan-Meier survival curve and log rank test. We investigated the possible prognostic factors for postoperative mortality (up to 30 days after surgery) with chi-square test and Fisher's exact test - in search for a connection between two quality valuables.

We used a critical level of significance of $\alpha=0.05$. The zero hypothesis is declined when the $P$-value is less than $\alpha$.

\section{Results}

Among the 155 patients included in the study, the male:female ratio was 1.76:1. The rate of new cases was highest $>65$ years.
When we excluded 54 patients who were receiving palliative treatment, among the remaining 101 we determined that TG had been performed more often - on 61 patients. In comparison, STG had been performed on 40 patents.

When we investigated other patient characteristics, which may influence patient survival such as age, sex, tumor differentiation, tumor localization, $\mathrm{N}$-stage, adjuvant therapy, and type of lymph node dissection, we figured out that the only significant factors were N-stage and D2 lymph node dissection (data not shown). First, we compared patients with TG and STG according to N-stage distribution (Table 1).

Table 1 shows that patient distribution in the two groups is very similar and patients with TG/STG can be compared independently of $\mathrm{N}$-stage or other patient characteristics.

Among the patients with $\mathrm{TG}$, four were localized in the cardia, 41 in the stomach corpus, and 15 in the pyloric antrum area. In comparison, among the patients with STG, the distally located tumors predominated -29 cases. Eleven were localized in the stomach corpus. In the "Supplementary materials" section, we have included photos of the microscopic appearance of the tumors (hematoxylin and eosin 20x, poorly cohesive carcinoma, Figure S1) and the performed operative interventions (Figures S2-S6).

When we compared the overall survival rate of both groups with total and subtotal resection, we could not determine any statistically significant difference $(P=0.824)$ as shown in Figure 3.

From the graph in Figure 3, we can see that in group 2 (STG), there is a certain tendency of better survival rate after the fourth year.

We also analyzed the perioperative mortality in the group of radically operated patients. Each death that occurred within 30 days of the operation was considered perioperative. Among the reviewed 101 radically operated patients, ten died within 30 days after the operation. This makes the perioperative death rate of this study $\sim 10 \%$. Half of the patients who died within 30 days after the operation underwent TG, the rest underwent subtotal resection. Despite that, we can safely assume that the volume of gastric resection in this study does not influence the perioperative death rate.

We also investigated other possible prognostic factors related to postoperative mortality within 30 days after surgery (ALB, total protein, splenectomy, and age). The

Table I Patient distribution according to $\mathrm{N}$-stage

\begin{tabular}{lllllll}
\hline N-stage & N0 (\%) & NI (\%) & N2 (\%) & N3 (\%) & Nx (\%) & Total \\
\hline Total gastrectomy & $20(32.8)$ & II (18.0) & $15(24.6)$ & $13(21.3)$ & $2(3.28)$ & 61 \\
Subtotal gastrectomy & $12(30.0)$ & $5(12.5)$ & $10(25.0)$ & $9(22.5)$ & $4(10)$ & 40 \\
\hline
\end{tabular}




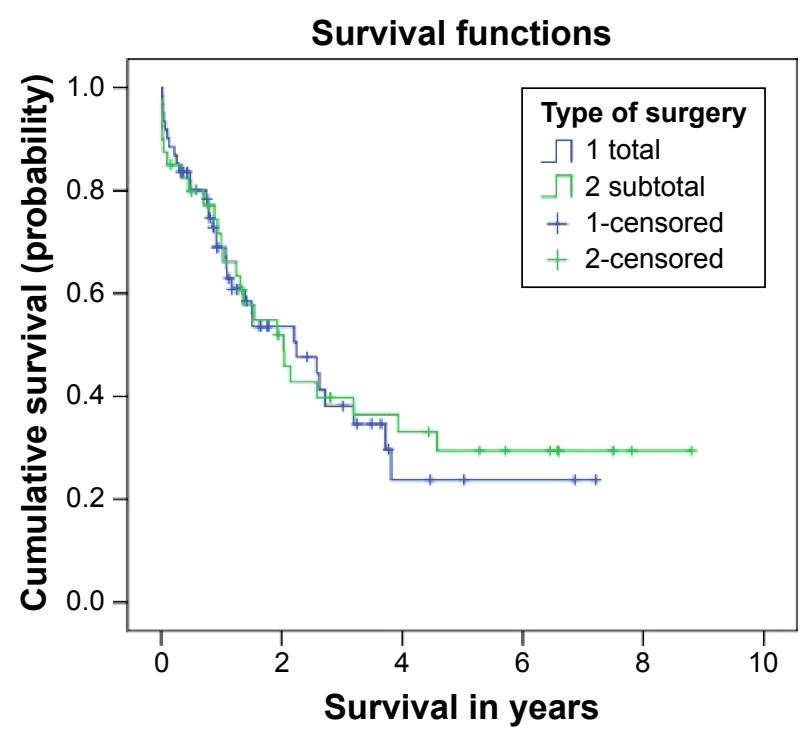

Figure 3 Overall survival rate of 101 patients based on the volume of gastric resection.

only significant factor was the age of the patient. Results are shown in Figure 4, $(P=0.026)$.

In order to eliminate the influence of the patients who were operated on in the last years of this study, we investigated the 3-year survival rate in both groups. The 3-year survival could be investigated in 66 patients, 36 with TG and 30 with STG. In the first group (the patients with TG), the 3-year survival rate was $37 \%$ and in the second group (those with STG) 39\%. The function of the survival rate and the risk is presented in Figure 5.

The resulting difference among the 3-year survival rates was insignificant, $(P=0.990)$.

In order to determine the influence of the type of radical operation, despite the type of lymph dissection (which is already a proven factor in the overall survival rate among the patients in this trial), we performed a statistical analysis

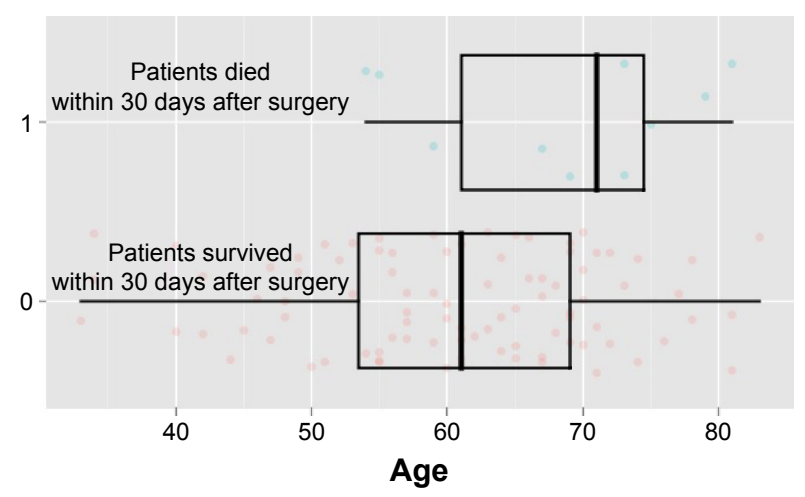

Figure 4 The comparison of postoperative mortality within 30 days after surgery according to average age of the patient.

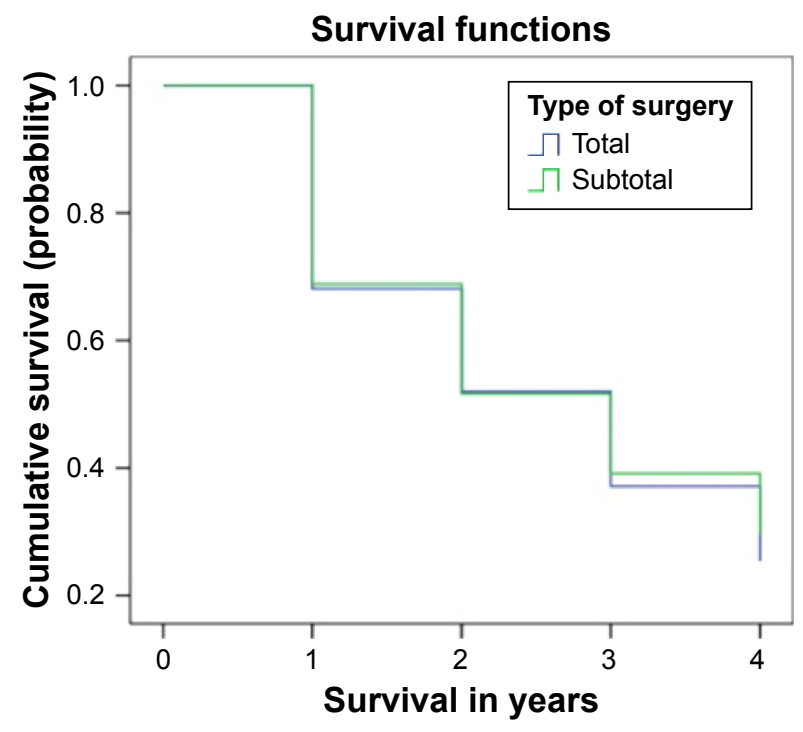

Figure $\mathbf{5}$ Three-year survival rate function according to resection type.

only on the patients with D2 lymph dissection based on the type of gastrectomy.

For this, we divided 60 out of the 101 radically operated patients into groups based on the gastric resection. The results are shown in Figure 6.

Again, we could not determine a statistically significant difference among the overall survival rate for both groups, $(P=0.787)$.

\section{Discussion}

The final conclusion of our study is that the volume of surgical resection has no influence on the survival of patients with

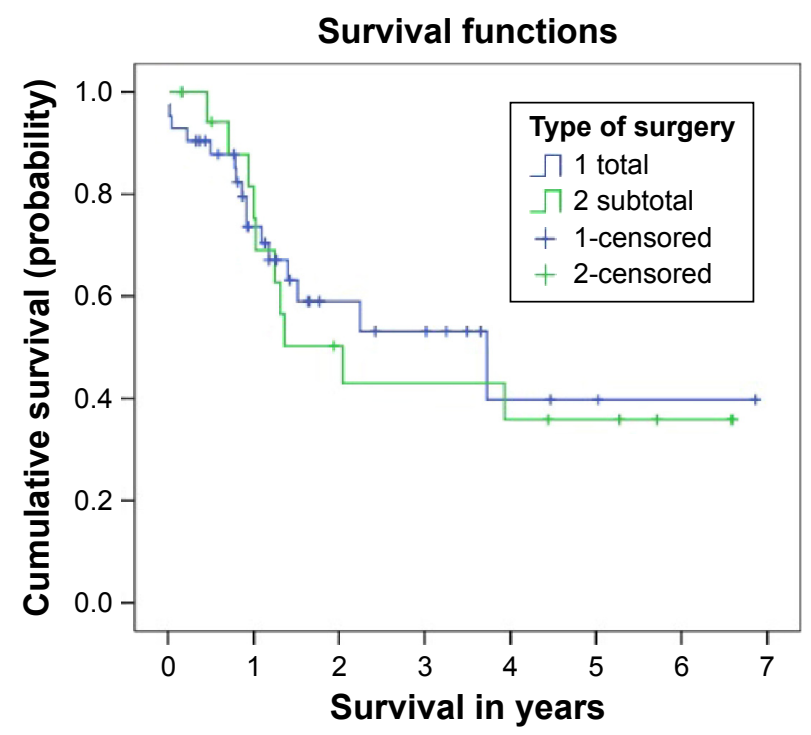

Figure 6 Overall survival of patients with D2 lymph dissection according to the volume of gastric resection. 
gastric cancer, irrespective of the performed lymph node dissection, or the N-stage of TNM classification. The only prognostic factor for postoperative mortality within 30 days after surgery was the patient's age at diagnosis. We cannot give preference to either surgical approach based on perioperative or postoperative survival. The decision regarding the volume of surgical resection in the current study is made by the surgeon based on tumor localization, differentiation, and patient's general status.

Several studies compare the efficiency, safety, and long-term survival rate of patients with gastric carcinoma based on the volume of stomach resection. Most of them either give preference to STG, especially for cancer of the distal stomach, or cannot find a significant difference when comparing the overall survival. The main disadvantage of TG is higher perioperative morbidity and mortality. Such results were found by Mocan et al in their study "Long term outcome following surgical treatment for distal gastric cancer", ${ }^{8}$ but we could not prove this in our study.

Another study of 338 consecutive gastric cancer patients showed a 5-year survival rate of $43 \%$ in patients after STG and $39 \%$ in patients with abdominal gastrectomy, without significant differences between the groups. In conclusion, the authors' silver lining is that the lower morbidity and mortality rate with a nearly identical long-term survival yielded by STG compared with TG leads them to justify STG, especially in elderly patients with comorbidity and a high operative risk, on condition that its performance is radical from an oncological point of view. ${ }^{9}$

In a study of 125 patients with radical gastric resection at Ewha Womans University Medical School, a comparative analysis was performed for the differences in clinicopathological characteristics and the prognosis between TG and STG. The 5-year survival rate for TG was lower (38.1\%) than STG (69.0\%). When tumor stages were stratified, there was no significant difference in the survival rate. ${ }^{10}$

A study in 2010 of 402 patients with middle-third advanced gastric cancer who underwent gastric resection analyzed the long-term prognosis according to the length of the proximal resection margin and the extent of gastric resection. The authors could not find any significant differences in the 5-year survival rates according to the length of proximal resection margin, but the 5-year survival rates of patients who underwent distal gastrectomy (DG) were significantly higher than the rates of the patients who underwent $\mathrm{TG}$ in curative cases ( $67.8 \%$ vs $58.4 \%, P=0.037)$. No significant difference in the stage-stratified survival rates according to the extent of gastric resection was observed. ${ }^{11}$
The results of our study demonstrate the lack of influence of the volume of the gastric resection on the long-term survival rate and the 3-year survival rate of patients with gastric adenocarcinoma. These results are similar to those of some other studies, which demonstrate that there is no survival benefit from a TG if resection margins are tumor free. ${ }^{12}$ Therefore, TG should only be performed by experienced surgeons and if the localization of the tumor requires that procedure.

We compared our results to two meta-analyses. The first was performed by Wen et al. Their meta-analysis includes one randomized controlled trial and seven retrospective studies involving 1,077 patients. The results showed no significant difference in 5-year overall survival rate (odds ratio $=0.89, P=0.53$ ). However, TG achieved a lower recurrence rate (odds ratio $=0.53, P=0.004$ ). TG experienced higher morbidity risk (odds ratio $=0.11, P<0.00001$ ). Operative mortality and nutritional states were comparable without significant differences. ${ }^{13}$ Similar results were reported in another meta-analysis from 2013, which investigated two randomized controlled trials and nine retrospective studies with a total of 1,364 patients. ${ }^{14}$

We mentioned patient age, ALB level, weight loss, and pancreatectomy as proven prognostic factors for postoperative mortality within 30 days after surgeries. Another study also found that metastatic disease diagnosed preoperatively or perioperatively, and poor tolerance of neoadjuvant therapy independently predicted postoperative mortality. ${ }^{15}$ The current study could only prove age as a prognostic factor, as the average age of patients who did not survive 30 days after surgery is higher than the average of those who did survive.

The volume of the lymph dissection is another controversial topic in the modern concept of gastric cancer. In order to remove the influence of lymph dissection on long-term survival rate, this study analyzed the survival rate based on the volume of gastric resection only in patients with D2 lymph dissection. The results concur that with clean resection lines, the volume of resection does not matter if the D2 dissection is performed in full volume. The current world practices of performing more organ-preserving operations are applicable in gastric cancer surgery. Here though, the clean resection lines matter the most.

This study has some limitations. Among them is the lack of criteria whether the gastric carcinoma should be operated on. According to the guidelines of the National Cancer Comprehensive Network, the gastric carcinoma is accepted as nonresectable if there is proof that the peritoneum 
is affected (including positive peritoneum cytology), distal metastasis or locally advanced disease (N3 or N4 lymph node subjected to imaging examination or proven by biopsy or invasion of the big vessels with the exception of the splenic vessels). The limited gastric resection should be performed even when the resection lines are positive for nonresectable tumors in palliative interventions. The drawback of this study is the lack of standardization of the resectability criteria. During the study years, those criteria had not been defined. Four different surgeons formed part of this retrospective study during the same time period. Each of them used their individual approach in the operative treatment. Another limitation of this study is the insufficient preoperative staging. This is due mainly to nationwide problems, despite the accepted standards for gastric cancer treatment in Bulgaria from 2009.

\section{Disclosure}

The authors report no conflicts of interest in this work.

\section{References}

1. World Health Organization [homepage on the Internet]. GLOBOCAN 2012: Estimated Cancer Incidence, Mortality and Prevalence Worldwide in 2012. WHO, International Agency for Research on Cancer; 2012. Available from: http://globocan.iarc.fr/Pages/fact_sheets_cancer.aspx. Accessed May 4, 2016.

2. sbaloncology.bg [homepage on the Internet]. Bulgarian National Cancer Registry, Cancer Incidence in Bulgaria 2011, TOM XXII, Volume XXII 2013. Available from: http://www.sbaloncology.bg/en/ncr.html. Accessed May 4, 2016.

3. Japanese Gastric Cancer Association. Japanese gastric cancer treatment guidelines 2010 (ver. 3). Gastric Cancer. 2011;14(2):113-123.
4. Kuo CY, Chao Y, Li CP. Update on treatment of gastric cancer. J Chin Med Assoc. 2014;77(7):345-353.

5. National Comprehensive Cancer Network. NCCN Clinical Practice Guidelines in Oncology (NCCN Guidelines). Gastric Cancer. v.3.2015. Available from: http://www.nccn.org/PDF/gastric.pdf. Accessed October 30, 2015.

6. Waddell T, Verheij M, Allum W, et al. Gastric cancer: ESMO-ESSOESTRO Clinical Practice Guidelines for diagnosis, treatment and follow-up. Ann Oncol. 2013;24 Suppl 6:vi57-vi63.

7. Bartlett E, Roses RE, Kelz RR, Drebin JA, Fraker DL, Karakousis GC. Morbidity and mortality after total gastrectomy for gastric malignancy using the American College of Surgeons National Surgical Quality Improvement Program database. Surgery. 2014;156(2):298-304.

8. Mocan L, Tomus C, Bartos D, et al. Long term outcome following surgical treatment for distal gastric cancer. J Gastrointestin Liver Dis. 2013; 22(1):53-58.

9. Gockel I, Pietzka S, Gönner U, Hommel G, Junginger T. Subtotal or total gastrectomy for gastric cancer: impact of the surgical procedure on morbidity and prognosis - analysis of a 10-year experience. Langenbecks Arch Surg. 2005;390(2):48-155.

10. Lee JH, Kim YI. Which is the optimal extent of resection in middle third gastric cancer between total gastrectomy and subtotal gastrectomy? J Gastric Cancer. 2010;10(4):226-233.

11. Jang YJ, Park MS, Kim JH, et al. Advanced gastric cancer in the middle one-third of the stomach: should surgeons perform total gastrectomy? J Surg Oncol. 2010;101(6):451-456.

12. Bozzetti F, Marubini E, Bonfanti G, et al. Subtotal versus total gastrectomy for gastric cancer: five-year survival rates in a multicenter randomised Italian trial. Italian Gastrointestinal Tumor Study Group. Ann Surg. 1999;230(2):170-178.

13. Wen L, Chen XZ, Wu B, et al. Total vs. proximal gastrectomy for proximal gastric cancer: a systematic review and meta-analysis. Hepatogastroenterology. 2012;59(114):633-640.

14. Pu YW, Gong W, Wu YY. Proximal gastrectomy versus total gastrectomy for proximal gastric carcinoma. A meta-analysis on postoperative complications, 5-year survival, and recurrence rate. Saudi Med J. 2013;34(12):1223-1228.

15. Robb WB, Messager M, Goere D, et al. Predictive factors of postoperative mortality after junctional and gastric adenocarcinoma resection. JAMA Surg. 2013;148(7):624-631. 


\section{Supplementary materials}

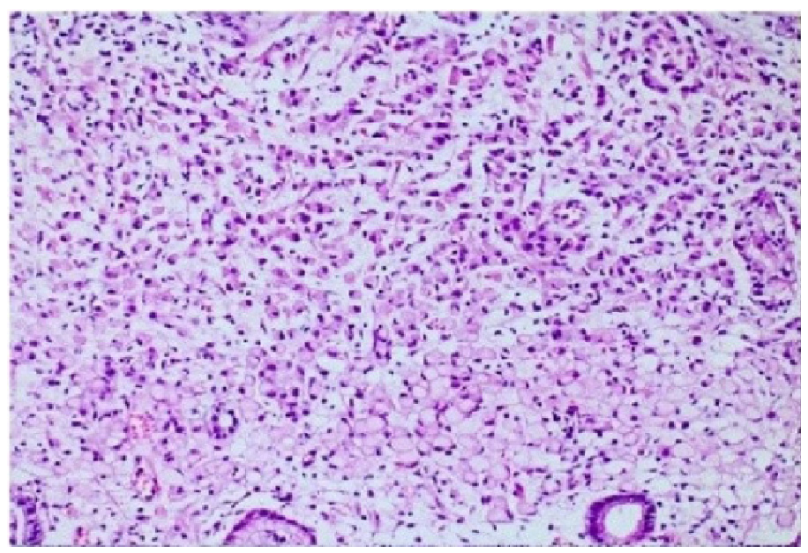

Figure SI Poorly cohesive gastric carcinoma.

Note: Hematoxylin and eosin $\times 20$.

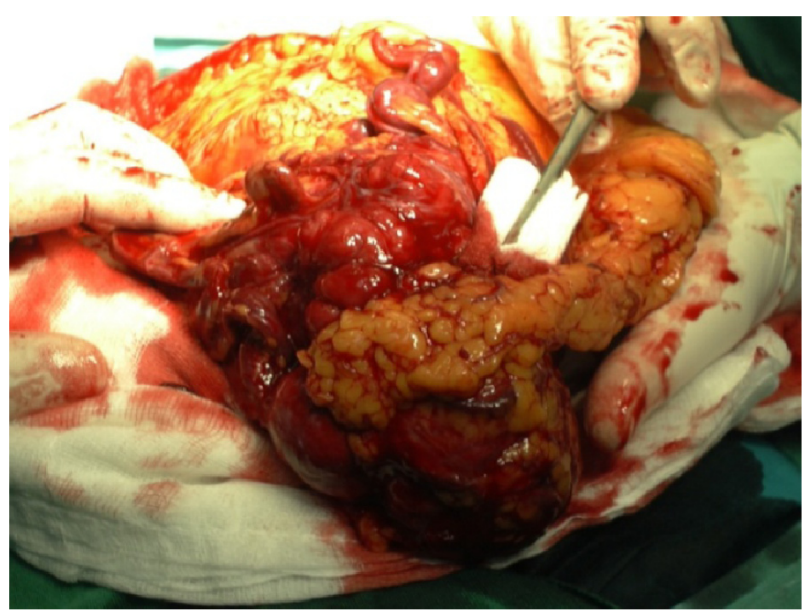

Figure S2 Patient with a huge gastric tumor (location - corpus).

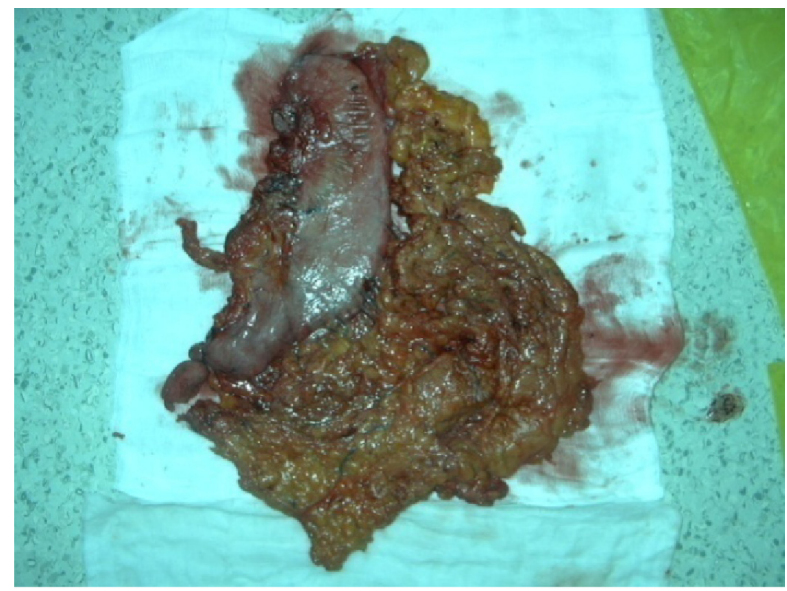

Figure S3 Operative preparation of a removed stomach with omentum.

Notes: The distal end is closed with a $5 / 5 \mathrm{~cm}$ lint. Tumor localization - in the area of angulus ventriculi and up to lesser curvature.

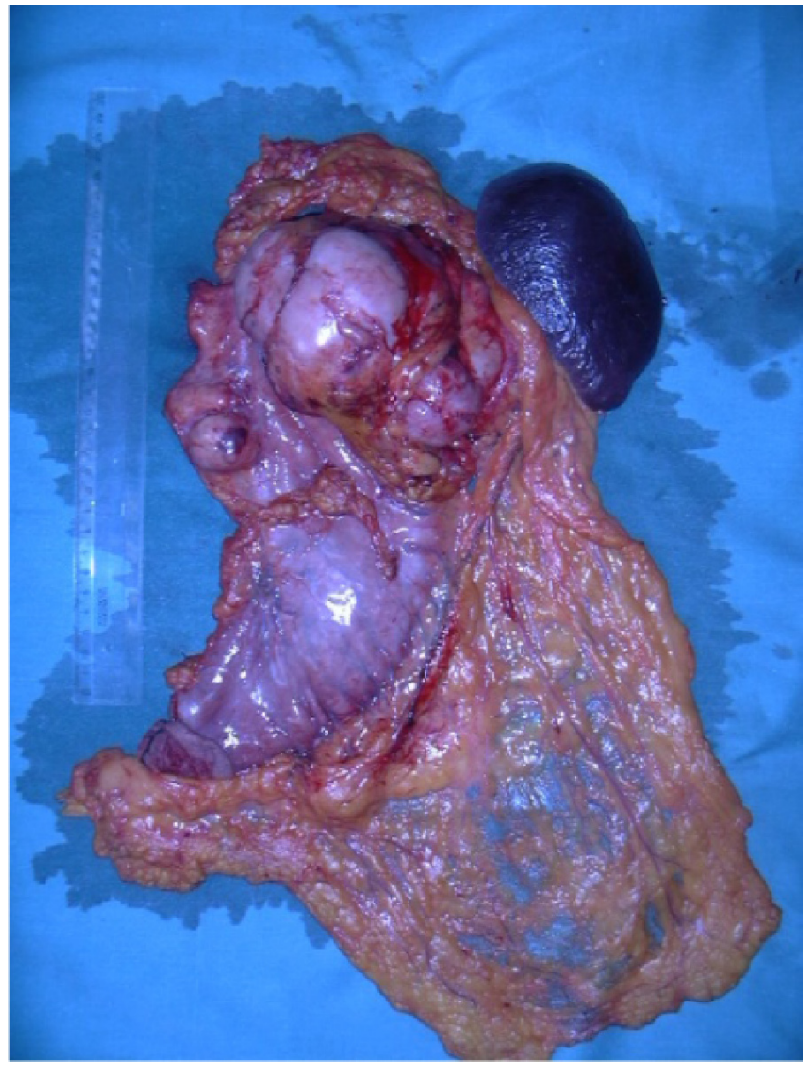

Figure S4 Gastrectomy, omentectomy, and splenectomy of a male patient with cancer with proximal localization - fundus-cardia- T4N2Mx.

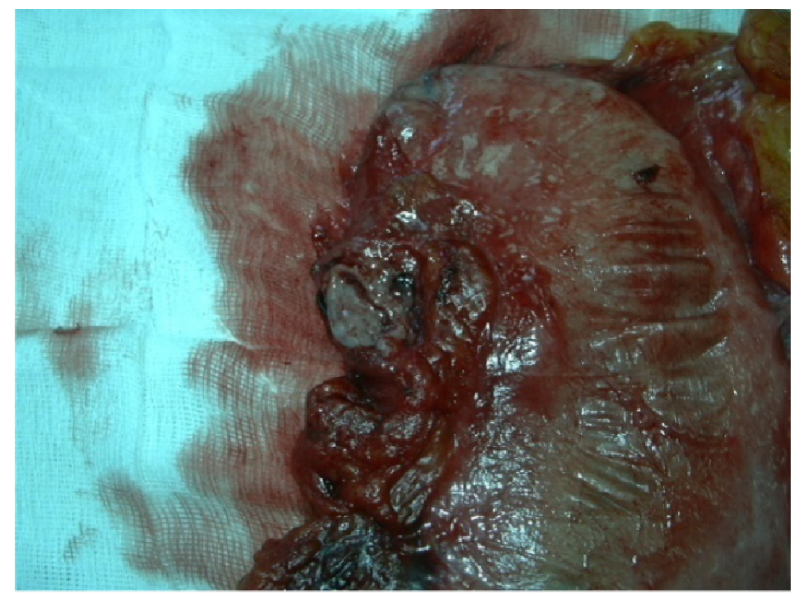

Figure S5 Part of the esophageal mucous.

Note: Visualizing the Z-line. 


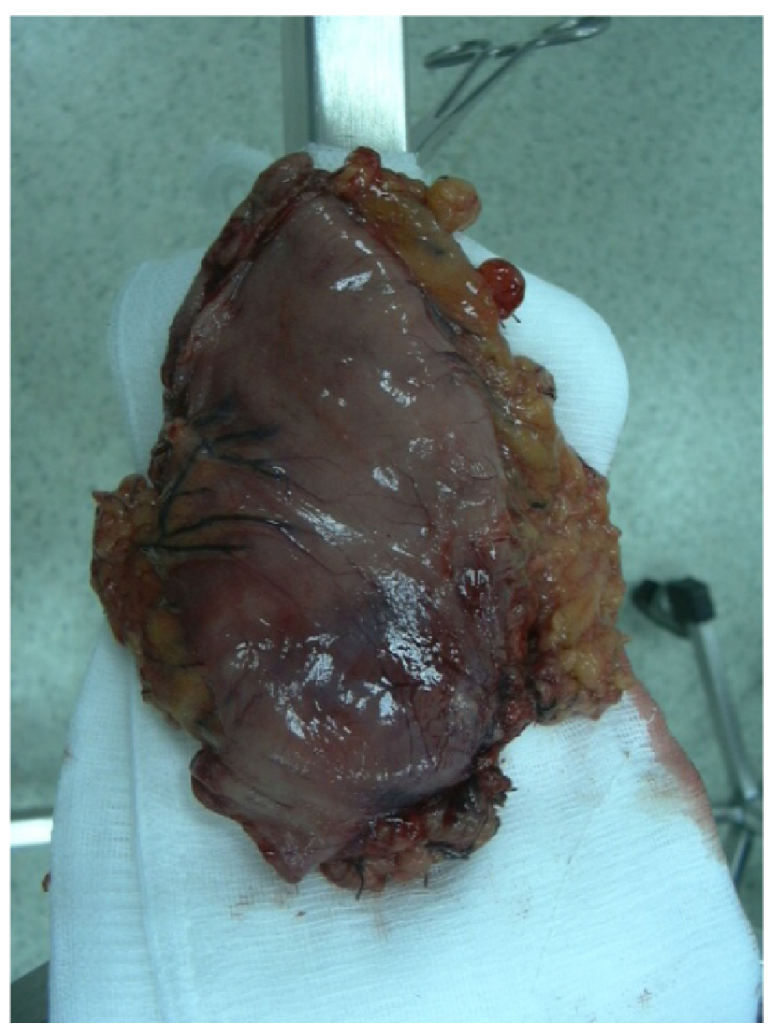

Figure $\mathbf{S 6}$ Subtotal gastric resection in a female with pyloric antrum localization of TINOMO cancer on the front stomach wall.

\section{Publish your work in this journal}

OncoTargets and Therapy is an international, peer-reviewed, open access journal focusing on the pathological basis of all cancers, potential targets for therapy and treatment protocols employed to improve the management of cancer patients. The journal also focuses on the impact of management programs and new therapeutic agents and protocols on patient perspectives such as quality of life, adherence and satisfaction. The manuscript management system is completely online and includes a very quick and fair peer-review system, which is all easy to use. Visit http://www.dovepress.com/testimonials.php to read real quotes from published authors. 